Archives

$42 \mid 2008$

Circulations et frontières

\title{
Introduction : la structuration des espaces et des frontières
}

\section{Maurice Aymard}

\section{OpenEdition}

Journals

Édition électronique

URL : http://journals.openedition.org/ccrh/3417

DOI : $10.4000 /$ ccrh.3417

ISSN : 1760-7906

Éditeur

Centre de recherches historiques - EHESS

Édition imprimée

Date de publication : 25 avril 2008

Pagination : 7-15

ISSN : 0990-9141

\section{Référence électronique}

Maurice Aymard, «Introduction : la structuration des espaces et des frontières », Les Cahiers du Centre de Recherches Historiques [En ligne], 42 | 2008, mis en ligne le 08 novembre 2011, consulté le 19 avril 2019. URL : http://journals.openedition.org/ccrh/3417 ; DOI : 10.4000/ccrh.3417

Ce document a été généré automatiquement le 19 avril 2019

Article L.111-1 du Code de la propriété intellectuelle. 


\title{
Introduction : la structuration des espaces et des frontières
}

\author{
Maurice Aymard
}

1 Pour remplir la mission qui m'a été confiée par les organisateurs de cette rencontre, j'ai longtemps hésité entre deux options. La première était de parler de Fernand Braudel luimême, et de la façon dont il a abordé et traité, aux différentes étapes de son œuvre écrite, le thème de l'espace, de ses structurations internes, des circulations qui l'animent et donnent vie à ces structurations, des frontières qui le divisent de manière temporaire ou durable. La seconde était de développer un exemple particulier, un objet relativement nouveau qu'il aurait rencontré sur sa route, qui aurait retenu son attention, mais dont il n'aurait jamais fait un objet central de ses recherches. Les deux pistes me tentaient de la même façon : aussi ai-je fait le choix qui m'est apparu le plus raisonnable, celui de ne pas choisir, et de les suivre l'une après l'autre.

Difficile en effet de ne pas nous référer d'une façon ou une autre à Braudel, dans le cadre d'une rencontre du Centre de Recherches Historiques (CRH), qu'il a fondé et dirigé, à propos du thème qui nous réunit ici. Mais à condition de préciser d'entrée de jeu que l'objectif n'est pas, ne peut pas être de réaffirmer la vérité d'un dogme face à tous ceux qui seraient tentés ou ont décidé de plus ou moins longue date de s'en éloigner ou d'en prendre le contre-pied pour mieux s'en libérer. Chacun est en effet libre de retenir de l'œuvre de Braudel, comme de toute autre, ce qui lui convient, en en rejetant ou négligeant le reste. Une seule obligation doit s'imposer : éviter de lui attribuer ce qu'il n'a jamais ni écrit ni pensé, du moins dans les termes que certains voudraient lui attribuer.

Dans cette perspective, une bonne précaution sera de se méfier de la fausse simplicité des textes de Braudel. Elle est le produit d'un long travail fait moins de corrections ligne après ligne que de réécritures répétées, de bout en bout, jusqu'à arriver à une rédaction qui le satisfasse. Car plus les réalités qu'il souhaite expliquer sont difficiles et complexes, plus il s'acharnera à simplifier la formulation de sa pensée. Mais plus aussi il se montrera attentif à ne pas se laisser enfermer dans telle ou telle de ses formules, et de se lier luimême les mains : chacune d'entre elles, dans la mesure même où il n'y voit que des 
approximations successives, des métaphores ou des images plutôt que des conceptualisations rigoureuses, va se retrouver, dans le même livre et d'un livre à l'autre (où les cartes se trouvent redistribuées), rééquilibrée, compensée, nuancée et relativisée par d'autres.

Il suffit de penser ici à l'expression d'économie-monde. Elle apparaît dès la première édition de la Méditerranée (1949) comme une traduction de l'allemand Weltwirtschaft, pour définir l'essence même de l'unité de la mer intérieure, qu'il propose d'identifier comme un monde non pas isolé, mais qui doit sa cohérence aux échanges économiques et aux circulations des marchandises, des hommes et des biens culturels qui s'y développent dans toutes les directions, et qui animent l'essentiel de sa vie économique. Malgré son rôle d'intermédiaire entre trois continents, la Méditerranée pourrait à la limite se suffire à elle-même, les échanges avec l'extérieur venant en quelque sorte en plus... Mais quand, trente ans plus tard, il réemprunte, dans le troisième volume, Le temps du monde, de Civilisation matérielle, économie et capitalisme (1979), ce terme à Immanuel Wallerstein qui l'avait appliqué dans son Modern World-System non plus à la Méditerranée mais à l'Europe, c'est pour désigner à son tour l'Europe telle qu'elle s'affirme au tournant du XvIe siècle dans sa capacité à étendre sa maîtrise des circulations maritimes à l'ensemble des océans et à mener à bien une première unification économique du monde. Mais il se hâte aussitôt de définir les limites de cette économie-monde européenne qui n'inclut entre XVI et XVIII siècle ni la totalité du continent, ni la totalité de la Méditerranée. L'espace russe, d'un côté, qui se constitue autour de l'axe qui va de la Caspienne à la Baltique et à la Mer Blanche, et, de l'autre, l'espace de l'Empire ottoman, présenté cette fois comme tournant le dos à la mer, et décrit comme un univers de caravanes qui traversent l'Anatolie et les Balkans, vivant jusqu'à la première moitié du xixe siècle de sa vie propre, et touché de façon marginale par les pressions de l'économie européenne qui s'arrêtent à ses frontières, se voient reconnaitre le statut d'économies-mondes autonomes que l'Europe ne réussira à intégrer, et encore, que tardivement, entre 1800 et 1850 . Et de toute façon, précise aussitôt Braudel, l'économie-monde, dans cette nouvelle définition, cette fois dynamique et non plus principalement statique comme dans la Méditerranée, n'est ellemême qu'un ordre parmi les autres, un niveau de lecture et de cohérence de l'organisation et des hiérarchies spatiales, mais non pas un ordre unique qui rendrait compte des autres.

De la même façon, dans Identité de la France, l'espace français pourra aussi être lu tour à tour comme structuré par la rencontre entre deux civilisations rurales, l'une, céréalière, venue de l'Est, et l'autre, méditerranéenne, venue du Sud et solidement installée par cinq siècles de domination romaine, et comme partagée, encore au XVIII siècle, entre une façade maritime ouverte sur les échanges à longue distance, et une moitié orientale terrienne qui tourne le dos à ces échanges. Ces deux couples d'oppositions, loin de s'exclure, se complètent, et visent l'un comme l'autre à mettre de l'ordre dans un espace placé sous le signe de la diversité. Car, souligne-t-il, et n'en déplaise aux lecteurs superficiels et pressés qui ont voulu voir dans ce livre écrit au soir de sa vie une forme de reniement, et une conversion tardive à un «identitarisme » contre lequel n'avait cessé de témoigner son œuvre antérieure : comme l'Europe, la France est diversité.

6 Multiplier les exemples de ce genre ne suffirait pas à nous garantir contre le risque de la simplification. Car ils ne prennent leur sens que dans le cadre de livres dont chacun répond à sa logique propre et cherche à porter un regard différent sur un ensemble ouvert de réalités historiques. Et ils appellent à chaque fois un très long, un trop long 
discours. Je limiterai donc mon propos à tenter d'expliciter ce que j'ai, sur le thème de notre rencontre, retenu de l'œuvre écrite de Braudel, et qui continue à me servir, consciemment ou non, de référence. Personnel par définition, ce choix n'en exclut, bien entendu, pas d'autres, tout aussi justifiés sans aucun doute, et peut-être préférables aux miens.

7 Les choses ne se comprennent bien qu'à une certaine distance : prendre un double recul, spatial et temporel, par rapport aux réalités observées est indispensable si l'on veut se protéger contre la myopie de qui reste collé à son objet d'étude. Braudel a pu ainsi écrire que le regard qu'il a porté sur la Méditerranée a bénéficié d'un double dépaysement. Le premier a été de la découvrir et d'apprendre à la voir depuis la rive sud - l'Algérie où, jeune agrégé, il avait été envoyé enseigner - c'est-à-dire "à l'envers » par rapport à la position la plus courante de l'observateur européen. Le second a été, pendant les trois années de son séjour au Brésil (1935-1938), de la voir de loin, de l'extérieur, à partir d'un monde qui était à sa façon une création de la Méditerranée, et un témoignage vivant et fascinant de son dynamisme. À ces deux dépaysements, il aurait pu sans peine en ajouter un troisième : celui de ses cinq années de prisonnier de guerre en Allemagne (1940-1945), pendant lesquelles, coupé de ses notes et de ses livres, il a rêvé la Méditerranée au point d'en faire le personnage central, le héros a pu écrire Paul Ricœur, de son livre. Ce « regard distancié » de l'historien répond à d'autres exigences que le "regard éloigné » de l'anthropologue : il ne vise pas à rompre la relation qui lie le chercheur à l'objet qu'il étudie, mais à voir cet objet à la fois dans sa totalité et sous un angle différent. Il ne se confond pas pour autant avec un quelconque point de vue de Sirius, qui effacerait par l'éloignement toutes les aspérités du relief : la Méditerranée du XVIe siècle ne devient réalité sous sa plume que parce que Braudel réussit à combler un vide, à inventer, à partir de sources pour leur très grande majorité occidentales, un partenaire ottoman à Philippe II, à imaginer la mer intérieure depuis Istanbul, et à croiser ainsi les regards. Ce qui ne condamne pas la monographie locale, bien au contraire : il a pu ainsi réutiliser dans l' Identité de la France son mémoire de diplôme sur Bar-le- Duc, et consacrer un chapitre au siège de Toulon de 1707. Mais elle n'est qu'une lecture parmi d'autres, à une échelle différente, de réalités et de représentations dont les détails prennent tout leur sens quand ils sont vus au plus près des acteurs, presque au ras du sol.

8 Les espaces n'existent comme tels que dans la mesure où ils sont construits par des circulations : celles des hommes, des marchandises, des biens culturels, ou encore des informations. D'où l'importance accordée, pour ces dernières, à Raguse et à Corfou, qui vivent à l'écoute des nouvelles en provenance de l'Empire ottoman, et notamment de ses préparatifs militaires, car le problème est chaque année de savoir à l'avance, si la guerre menace, l'importance et la destination de la flotte qui sortira en avril de l'Arsenal d'Istanbul : d'un côté des informateurs, espions, voyageurs ou marchands, ou les trois à la fois; de l'autre des circuits assurant l'acheminement rapide de ces nouvelles tant attendues mais toujours incertaines en direction de Naples, Gênes et Venise, de l'Escorial et de la Cour de France, là où elle se trouve. Vues de la terre, les circulations qu'il aura tendance à privilégier seront en revanche celles des hommes (les migrations montagnardes), des animaux (les différentes formes de la transhumance) et des produits (blé, huile et vin, fromages, bétail et bois), entre les trois étages de l'écosystème méditerranéen: plaines, collines et montagnes, reliées entre elles par des échanges réguliers, mais soumis aux multiples aléas de la conjoncture. Vues de la mer, ces circulations lui permettront de mettre en cause tantôt la totalité de la Méditerranée, 
tantôt les différents sous-ensembles qui la composent, comme l'Adriatique ou la Mer Egée, tantôt les mondes insulaires refermés sur eux-mêmes ou bien ouverts sur les échanges à très longue distance. Mais le mérite des Méditerranéens reste à ses yeux d'avoir progressivement construit les routes et les règles de la navigation (fondées sur la connaissance des vents et des courants) dans l'Atlantique central et dans l'Atlantique méridional (où, pour ce dernier, les Portugais ont joué, comme l'a montré V. Magalahaes Godinho, un rôle décisif), puis en partie aussi dans l'Atlantique nord , qui prépareront et rendront possibles les voyages de Colomb, de Vasco de Gama et Cabral, de Cabot et Verazzano : autant de systèmes particuliers de circulations régulières, portant encore sur un nombre réduit de navires, de marchandises et d'hommes, mais suffisants pour lier durablement le destin du nouveau continent à notre ancien monde.

Ces espaces sont souvent (mais pas toujours) des réalités historiques de longue durée. Une durée plus longue encore que celle des civilisations qui les occupent aujourd'hui, et, à plus forte raison, que les religions qui se sont « saisies de ces civilisations » : l'affirmation, formulée dans son manuel sur Le monde actuel (1963), réédité en 1987 sous le nom de Grammaire des Civilisations, vaut en premier lieu pour les pays du Proche et du Moyen Orient, où l'invention de l'écriture a accompagné la formation des premiers États centralisés, dotés d'une bureaucratie et d'une armée permanentes, et des premières villes.

10 Ces espaces sont des espaces-temps, impossibles à étudier et comprendre indépendamment des systèmes techniques qui en règlent les circulations : chameaux, dromadaires et mules, galères et bateaux ronds, marciliane et simples barques qui longent les côtes de l'Adriatique ou la traversent. Ces systèmes techniques lui permettent de définir une Méditerranée de quarante jours. Celle-ci ne peut être, elle aussi, qu'un niveau supérieur, et elle laisse toute leur place à d'autres niveaux spatiaux autour desquels s'organise pour l'essentiel, et doit donc se lire la vie quotidienne des hommes. Mais tel est son pouvoir d'attraction que certains de ces derniers se laissent tenter par l'aventure, comme ces renégats calabrais qui fuient leur village pour aller chercher fortune dans un Empire ottoman qu'ils rêvent comme plus accueillant.

11 Les frontières ne sont pas, dans cette perspective, premières, mais secondes. Elles sont le produit des dynamiques qui structurent ou ont, plus souvent encore, structuré dans le passé ces espaces: elles deviennent alors des réalités de longue durée, suffisamment intériorisées dans les esprits pour en faire oublier leurs origines lointaines. Tel est le cas, à l'échelle de la Méditerranée, de la frontière entre monde hellénistique et monde latin, reprise et institutionnalisée par l'Empire romain dont Paul Veyne a rappelé récemment avec force qu'il était un empire bilingue : c'est elle qui a séparé pendant tout le second millénaire les chrétientés d'Occident et d'Orient, et la récente guerre de Yougoslavie nous a rappelé qu'elle gardait aujourd'hui encore toute sa force. Mais, dans la mesure même où elle a été prolongée jusqu'à la Baltique par la dynamique de la christianisation de notre continent, elle a aussi divisé durablement l'Europe, tout comme le limes romain a séparé deux Europes, la première qui est restée fidèle à Rome au moment de la Réforme, la seconde qui en a remis en cause l'autorité - une autorité qu'elle n'avait jamais vraiment acceptée. Comme pour Marc Bloch dans son histoire des paysages ruraux, les frontières se situent pour Braudel à la fois au-delà et en deçà du politique, dans la mesure même où elles s'inscrivent dans une durée différente, infiniment plus longue, et mettent en cause tout un ensemble de réalités sur lesquelles le politique n'a eu, jusqu'à une date très récente, aucune prise. Ce qui ne veut pas dire que les institutions et le politique n'aient 
joué aucun rôle dans leur définition et leur stabilisation. Même contesté par François $1^{\text {er }}$, le traité de Tordesillas - un pari sur un avenir virtuel - a préparé une frontière durable entre Amérique espagnole et portugaise. Et, en France, le Rhône a continué à jouer pendant des siècles son rôle de frontière. Mais les frontières qui, au fond, intéressent le plus Braudel, héritier d'une longue tradition de la géographie humaine, sont celles qui sont inscrites dans le sol et le paysage avant même de l'être dans les consciences, et acceptées comme des évidences : les frontières des plantes cultivées, des formes des champs, des fonds de cuisine, des systèmes alimentaires. Et, bien entendu, les frontières de civilisation.

12 Mais ces mêmes frontières ne l'intéressent aussi que parce qu'elles ne constituent pas des absolus : elles conditionnent les circulations sans jamais ni prétendre ni réussir à les interdire totalement. Les voyages des biens culturels lui en fournissent des poignées d'exemples :

[...] voyages des hommes, voyages des biens aussi, des biens culturels, les plus usuels comme les plus inattendus. Ils ne cessent de se déplacer avec les hommes eux-mêmes. Apportés ici par les uns cette année, repris par d'autres l'année suivante ou un siècle plus tard, on les voit sans cesse transportés, abandonnés, ressaisis, et par des mains parfois ignorantes [...] $]^{1}$.

13 Ces circulations justifient sa conclusion sur les « rayonnements, emprunts mais aussi refus d'emprunter » par lesquels les civilisations se définissent les unes par rapport aux autres. Cette dernière citation, après d'autres, devrait suffire à évacuer du débat la vieille accusation de déterminisme géographique, souvent reprise contre Braudel : il suffit de se reporter à la première page de la Méditerranée. L'unité physique de l'espace méditerranéen, ce sera "le climat et les hommes ", l'unité humaine ce sera "les routes et les villes ", donc à nouveau les hommes. Mais à chaque fois tous les hommes : c'est-à-dire les générations antérieures, dont les choix répétés conditionnent et limitent nos propres choix. Les espaces fonctionnent, à leur façon, autant et plus encore que les frontières, comme des "prisons de longue durée » : des prisons édifiées elles aussi par les hommes eux-mêmes.

14 À ce choix de formulations et d'idées empruntées aux textes braudéliens, je serai tenté d'ajouter la remarque de Denys Lombard (dont Le Carrefour javanais a été sans doute, comme l'avait noté Bernard Lepetit, le dernier grand livre d'inspiration braudélienne) sur les deux représentations de l'espace, qui distinguaient selon lui les historiens de l'Europe de ceux de l'Asie : celle, plus familière aux premiers et plus courante sous leur plume, de l'espace comme continuité, identifiée en fin de compte avec des territoires, dont les limites idéales coïncident avec des frontières politiques et administratives, récentes ou plus anciennes; et celle, qu'il défendait lui-même à partir de sa connaissance de l'Asie du sud-est, de l'espace comme discontinuité, structurée par des réseaux reliant entre elles, à longue et très longue distance, des villes. La logique même de la Méditerranée a sans aucun doute conduit Braudel à privilégier lui aussi la première de ces deux représentations, ce qui explique que son éditeur italien, Einaudi, ait pu lui imposer de modifier le titre de son livre pour en faire «États et Empires dans la Méditerranée » de la seconde moitié du XVI siècle. Mais les observatoires qu'il s'est choisis dans un second temps, après ses premiers séjours à Simancas et à Madrid - Gênes, Venise, Raguse notamment - lui ont permis de pressentir la seconde : on la retrouve au cœur de ses définitions, en 1979, de l'économiemonde européenne, dont le centre est toujours à ses yeux une ville, en rapport avec d'autres villes, qui sont à la fois ses concurrentes et ses partenaires indispensables - et cette ville est elle-même toujours un port. Les espaces sont donc en fin de compte, autant 
sinon plus que des constructions historiques, des constructions des historiens : ce qui explique que Braudel ait, pour retrouver sa liberté, joué sur plusieurs espaces différents (la Méditerranée, le monde, l'Europe, la France), diversifié les échelles d'analyse, et cherché à mettre en évidence - à l'image du modèle d'organisation hiérarchisée des marchés, repris de Cantillon, et dont la base est, avec le bourg, le marché local - la façon dont ces différents espaces s'emboîtent et interfèrent les uns avec les autres.

L'exemple de l'esclavage, auquel nous avons consacré, Jean-Yves Grenier, Gilles PostelVinay, Alessandro Stanziani et moi-même, un séminaire de deux ans (2003-2005), me servira ici pour tester l'utilité actuelle de ces différentes propositions :

- première constatation: le terme d'esclavage regroupe en fait, sous un terme unique, une multiplicité de situations caractérisées par l'infériorité juridique statutaire de groupes plus ou moins nombreux d'êtres humains, mais souvent très différentes les unes des autres, au point que la traduction par un terme unique (esclave ou slave) les termes qui servent à les désigner dans les langues locales apparaît au minimum discutable, et souvent abusive. Le fait est particulièrement évident pour l'Afrique subsaharienne, qui a constitué pendant un bon millénaire, et sans doute pendant plus longtemps encore, le principal centre mondial d'exportation d'esclaves à longue distance: les chercheurs, après avoir longtemps refusé ou évité de reconnaître l'existence et l'importance d'un esclavage local, ont été tentés de plaquer sur ce dernier les contenus juridiques et sociaux de l'esclavage européen, pour se résoudre enfin à un inventaire précis des termes utilisés et des situations concrètes des individus concernés. Mieux vaudra donc parler, au pluriel, " d'esclavages ", distinguer les espaces où ont été enregistrées et observées ces situations, et en préciser avec soin la chronologie au lieu de leur prêter a priori une permanence qui est loin d'avoir toujours été la leur. Dans l'espace comme dans le temps, l'esclavage est diversité. Il est loin d'être une catégorie universelle, et il renvoie toujours à une histoire ;

- seconde constatation : les analyses de l'esclavage restent aujourd'hui encore tributaires de sa conceptualisation par les Européens à partir de leur propre expérience historique, qui s'organise autour de deux moments forts, chacun localisé dans son espace particulier. Le premier est celui de l'Antiquité classique, où la Grèce et Rome, dans lesquelles notre tradition culturelle a voulu situer les origines des valeurs de la civilisation et de l'histoire européennes, ont largement pratiqué l'esclavage comme la plupart des sociétés organisées de la Méditerranée de l'époque : elles ont servi de base à l'élaboration du mode de production esclavagiste comme étape historique du développement des sociétés. Le second a été celui, entre XVI ${ }^{e}$ et XIX siècle, de la traite transatlantique organisée de façon massive par les Européens pour les besoins de l'économie de plantation qu'ils avaient introduite dans le Nouveau Monde dans les décennies qui ont suivi sa découverte, pour faire face au déficit de main-d'œuvre provoqué par l'effondrement des populations locales: le recours à des esclaves importés de l'extérieur contredisait alors l'évolution générale observée à la même époque en Europe occidentale, qui allait plutôt dans le sens d'une généralisation du travail libre et rémunéré.

Les sociétés européennes, en décidant, dans la première moitié du XIX siècle, d'interdire la traite, puis d'abolir l'esclavage lui-même, avaient pris l'initiative de mettre un terme à ce paradoxe, et d'écrire ainsi ce qu'elles croyaient être le troisième et dernier chapitre de cette histoire de longue durée, dont les deux premiers semblaient d'ailleurs totalement déconnectés l'un de l'autre: n'étaient-ils pas séparés par un millénaire pendant lequel l'Europe médiévale semblait avoir rompu, non sans mal ni résistance d'ailleurs, avec l'esclavage chez elle? L'interdiction de réduire des Chrétiens en esclavage n'avait-elle pas 
créé une situation où les esclaves ne pouvaient désormais être que «les autres, chez les autres et pour les autres»? Donc une catégorie presque résiduelle, sinon marginale, même si les marchands occidentaux n'avaient pas hésité, entre XIII et $\mathrm{XV}^{\mathrm{e}}$ siècle, à se livrer à un commerce particulièrement profitable, à partir notamment de la Mer Noire, pour approvisionner en esclaves la Méditerranée musulmane. Et même si les flottes chrétiennes de galères avaient au XVIe siècle systématiquement mis à la rame leurs prisonniers de guerre musulmans, avant de trouver dans les condamnés des tribunaux une main-d'œuvre abondante et infiniment moins coûteuse ;

19 - troisième constatation : du même coup, identifier ailleurs, dans d'autres sociétés, pour les étudier de l'intérieur, d'autres pratiques et d'autres utilisations, générales ou limitées, de l'esclavage, n'a pas constitué jusqu'à une date récente, une priorité de la recherche. Il a fallu attendre le début des années soixante-dix pour voir cette situation changer peu à peu, tant à propos de l'Afrique que de la traite transsaharienne à destination des pays musulmans. Le déplacement du regard, le choix de nouveaux lieux et angles d'observation, ont permis un indiscutable développement des recherches et contribué à renouveler les problématiques et les questions posées au passé. La capture et le commerce des esclaves ont pris leur place dans l'histoire des sociétés africaines, avec l'identification de leurs principaux acteurs, guerriers et marchands, ainsi que des États qui ont tiré de ces activités une part croissante de leurs ressources. La place, les rôles et les statuts des différentes catégories de prisonniers que l'on continue à regrouper sous le nom d'esclaves, ont fait l'objet d'enquêtes approfondies, tout comme l'incapacité de l'abolition de l'esclavage à abolir la marque que celle-ci avait imposée sur les individus concernés et sur leurs descendants, et qui se maintient en fait pendant plusieurs générations. Et surtout un espace nouveau s'est imposé dans les recherches: le monde musulman de la Méditerranée et du Moyen-Orient.

Celui-ci a été le pôle principal du trafic des esclaves africains et son destinataire presque exclusif pendant la première moitié du second millénaire, l'un de ses deux pôles entre XVII et XIXe siècle, le dernier débouché des trafics partis du sud du Sahara entre mi-XIXe et les années vingt, et enfin le dernier bastion de l'esclavage jusqu'à aujourd'hui (Maurétanie et péninsule arabique). Cet espace s'est progressivement élargi le long des côtes de l'Océan Indien, de l'Afrique Orientale à l'Inde. Nous en connaissons désormais les limites temporelles. Et nous savons mieux également que cet espace, loin d'être homogène, a été en fait partagé entre plusieurs civilisations esclavagistes : celles-ci n'ont pas fait des esclaves le même usage (eunuques, esclaves domestiques, préférences affichées selon les lieux et les époques pour les esclaves masculins ou féminins, plantations comme dans l'Irak des VIII et IXe siècles pour le sucre, et à Zanzibar au XIXe pour le clou de girofle, esclaves du palais, esclaves-soldats, esclaves-ministres). Et elles n'ont pas renoncé à l'esclavage au même moment et dans les mêmes conditions.

21 Cet inventaire des situations relevant de près ou de loin de ce qu'il est convenu d'appeler l'esclavage est loin d'être aujourd'hui terminé. D'autres es-paces, qui n'ont été qu'à peine abordés, restent à interroger. Et l'historiographie européenne est loin encore d'avoir renoncé au monopole qu'elle avait réussi à imposer sur ce thème : un bon exemple en est fourni par le tour pris par les débats récents sur la comparaison des chiffres des traites transsaharienne et transatlantique, comme si la valorisation de la première pouvait contribuer à déculpabiliser l'Europe. Resterait enfin à établir le pont avec tous les travaux, eux aussi récents, sur "l'esclavage moderne », placé sous le signe du fait bien plus que du droit : derrière sa progression rapide, régulièrement dénoncée, il faut voir le 
rôle nouveau assumé par les frontières, cette fois comme barrières politiques imposées et surveillées avec une efficacité croissante par les États. Leur franchissement fait basculer dans la clandestinité et dans un statut de non-droit un nombre croissant de travailleurs migrants, que le coût du voyage et du passage pousse par ailleurs à des formes d'esclavage pour dettes, en principe temporaire. Et les obstacles opposés par les États à la mobilité de ces migrants contribue à faire basculer vers le travail dépendant chez eux les candidats potentiels ou malheureux au voyage. Plus que jamais l'espace reste une clef de lecture nécessaire du monde social : la leçon braudélienne n'a rien perdu de son actualité.

\section{NOTES}

1. Fernand Braudel, La Méditrranée et le monde méditerranéen à l'époque de Philippe II, Paris, A. Colin, 1949 (1 1 ère édition), p. 555-560.

\section{AUTEUR}

\section{MAURICE AYMARD}

MSH/Paris 